\title{
Compact 3D-Printed Circularly Polarized Antenna for Handheld UHF RFID Readers
}

\author{
Riccardo Colella, Member, IEEE, Andrea Michel, Member, IEEE, Luca Catarinucci, Member, IEEE
}

\begin{abstract}
In this work, the aptness of the combination of 3D-printing and RFID is faced by presenting a compact, low-profile, and cost-effective circularly polarized antenna for handheld UHF RFID reader. The radiating element has been realized through a circular array of four inverted-F monopoles, where the array elements are excited with a 90-degree phase offset through a microstrip feeding network, realized in 3D-printing technology as well. Taking advantage from low losses and moldability of the 3D-printing materials joint to a specific design strategy, the proposed antenna realizes an improved gain and an appreciable size reduction if compared with the state of the art.
\end{abstract}

Index Terms - Circularly polarized Antennas, 3D printing, Energy Efficiency, RFID Reader, UHF.

\section{INTRODUCTION}

$\mathrm{P}$ assive UHF radiofrequency Identification (RFID) in UHF band $(860 \div 920 \mathrm{MHz})$ is growing at a rapid pace and covers many different types of applications. At the same time, additive manufacturing 3D-printing technology promises high added value in many fields, including electromagnetics [1]-[3]. In such a context, the combination of 3D-printing and RFID could enable the design of new and challenging antennas more and more efficient other than compact. Emblematic in the literature is the case of antennas for handheld UHF RFID readers where stringent requirements, not only in terms of gain, polarization, and bandwidth, but also in terms of shape, weight, and size must be necessarily respected. Generally, these antennas are realized in planar technology by using either common materials (i.e. FR-4 substrates) or hi-performing RF laminates (i.e. Rogers) [4] so to exploit the lower losses to make the antenna compact while guaranteeing and adequate gain. In particular, in Fig. 1, solutions proposed in literature [4]-[16] have been classified as a function of both gain and occupied area. As clear from the graph, most of the antennas having a positive gain exhibit considerable size, larger than $\lambda / 4 \times \lambda / 4$. The work presented in [7] and [6] are exceptions. In particular, the antenna proposed in [6] consists of two crossed dipoles properly fed to obtain a circularly polarized antenna. However, in this solution the ground plane is not taken into account, which is indeed helpful to alleviate the effect of the presence of the human hand and electronic circuitry. On the other hand, the antenna presented in [7] is compact while guaranteeing a good gain (Fig. 1), and it can be seen as an example of transition from planar to 3D topology in the framework of antennas for handheld RFID readers. In fact, two layers of a low-loss dielectric material $\left(\varepsilon_{\mathrm{r}}=4.3, \tan \delta=0.002\right)$ opportunely and precisely separated have been used to take advantage from the third dimension. Interesting results in terms of both gain and size have been obtained, with prospective of further improvements, as for example the dual band capability introduced in [17]. In such a context, additive manufacturing 3D printing represents a real added value as it enables the possibility to control the dielectric characteristics of the substrate by accurately setting the percentage of released material (infill percentage) [3],[18].

In this work, low deformability, good thermal stability, robustness, and very low electromagnetic losses of the Polilactic Acid (PLA) at the considered frequencies have been exploited to 3D-print a square circularly polarized antenna as compact as $\lambda / 6 \times \lambda / 6 \times \lambda / 40$ and easily embeddable in commercial UHF-RFID (ETSI UHF band, 865-868 MHz) portable readers. The antenna consists of a 3D-printed PLA structure, opportunely characterized in terms of permittivity and loss tangent, which hosts an array of four inverted-F monopoles on the top layer. These radiating elements are excited with a $90^{\circ}$ phase offset through a microstrip feeding network placed on the bottom side of the structure.

Once designed and realized, reflection coefficient, isolation parameter and axial-ratio (AR) have been measured and obtained results have been discussed and compared with those available in literature. 


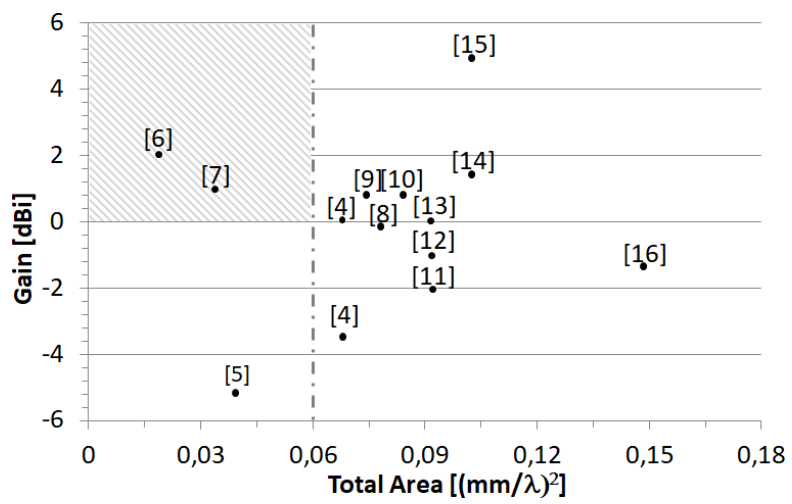

Fig. 1 Classification of antennas for handheld UHF RFID Readers in terms of size and gain. The size is normalized to the free space wavelength at the central frequency of the operating band.

\section{ANTENNA DESIGN}

In order to design the novel compact antenna for handheld UHF RFID readers based on 3D printing materials, preliminary measurements aiming at characterizing the PLA from the electromagnetic point of view have been performed. In particular the relative dielectric constant and loss tangent of PLA substrates when varying the infill percentage from $20 \%$ to $100 \%$ have been measured through the T-resonator method [18]. Results at $866 \mathrm{MHz}$ have been summarized in Tab. I. As can be observed, permittivity increases with the substrate density. Since losses are small enough in each investigated situation, the $100 \%$ case has been considered in this project, with $\varepsilon_{\mathrm{r}}=2.57$ and a loss tangent of only $\tan \delta=0.0069$. Indeed, the higher dielectric permittivity achievable with the $100 \%$ infill percentage is useful to further miniaturize the radiating element, allowing for a more compact overall structure.

TABLE I.

MEASURED PLA DIELECTRIC PARAMETERS AT 866 MHZ BY VARYING THE INFILL PERCENTAGE

\begin{tabular}{ccc}
\hline \hline Infill Percentage & Dielectric Constant & Loss Tangent \\
\hline $\mathbf{2 0 \%}$ & 1.49 & 0.0029 \\
$\mathbf{4 0 \%}$ & 1.62 & 0.0036 \\
$\mathbf{6 0 \%}$ & 1.91 & 0.0047 \\
$\mathbf{8 0 \%}$ & 2.23 & 0.0052 \\
$\mathbf{1 0 0 \%}$ & $\mathbf{2 . 5 7}$ & $\mathbf{0 . 0 0 6 9}$ \\
\hline \hline
\end{tabular}

The proposed 3D-printed antenna, designed to operate in the ETSI UHF-RFID band (865-868 MHz), is composed of two parts sharing the same ground plane: a set of four radiating elements disposed to form an annular square structure (Fig. 2a), and a microstrip feeding network (Fig. 2b). As clear from Fig. 2a, each radiating element is an inverted-F monopole with a capacitive

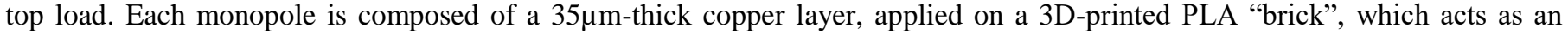
antenna substrate integral with a single support plastic structure. The capacitive top load is effective in reducing the physical size of the radiating element, thus contributing - along with the 3D-printable structure - on guaranteeing a more compact and lowprofile structure. Moreover, the thickness of the substrate of each inverted-F monopole is considered as a tunable parameter to effectively control the antenna gain while optimizing the occupied area. Specifically, the lower is the inverted-F monopole height, the more compact is the overall antenna structure, but the lower is the radiation efficiency. The radiating structure is completed with lateral feeding lines providing the connection with the feeding network. In the inset of the same Fig. 2a, the detail of the connection line of a single monopole is highlighted along with the related length parameters. In particular, the two parameters related to the short-circuited stub, $s_{l}$ and $s_{2}$, are also optimized to adjust the radiating element input impedance, which is equal to $100 \Omega$. The structure has been designed and simulated in CST Microwave Studio where an ad-hoc virtual material having the dielectric characteristics of the 100\%-PLA (see Tab. I) has been defined to reproduce the 3D printable parts of the antenna. With the optimized antenna parameters reported in Tab. II a total size of $60 \times 60 \times 7.5 \mathrm{~mm}^{3}$ has been obtained.

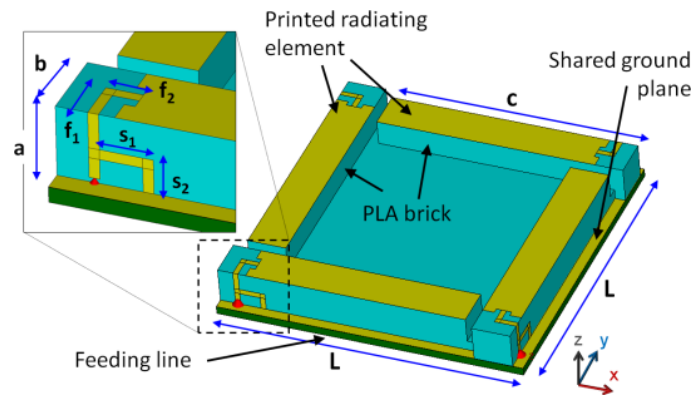

a) 


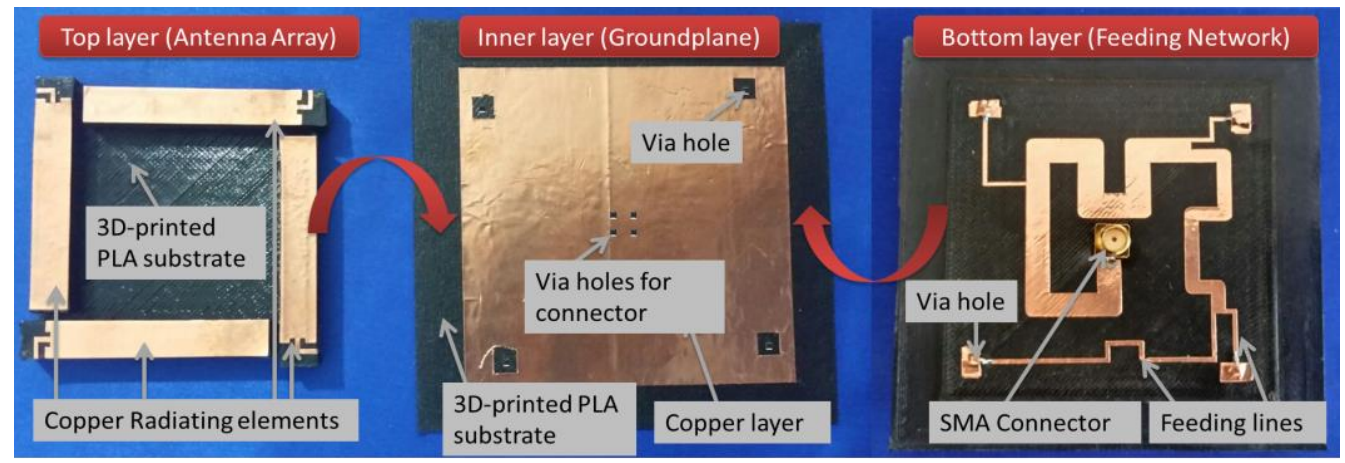

Fig. 3 Components of the designed 3D-printed antenna for handheld UHF RFID Reader
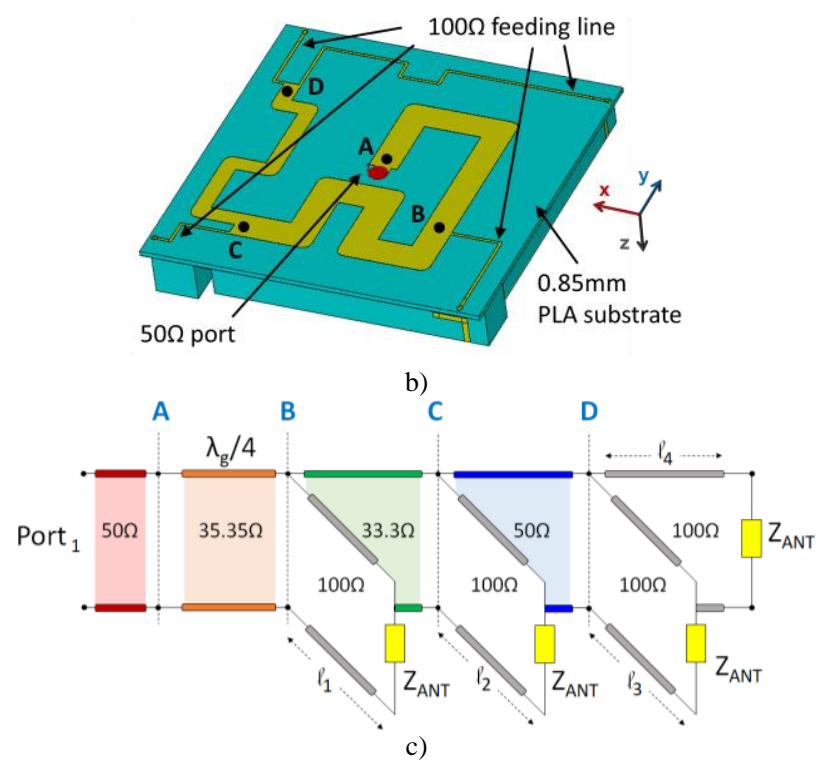

Fig. 2 Antenna layout: (a) 3D view and (b) bottom view. In (c), the equivalent circuit of the matching and delay network shown in (b) is shown, where $\mathrm{Z}_{\mathrm{ANT}}=100 \Omega$.

TABLE II

3D-PRINTED ANTENNA PARAMETERS

\begin{tabular}{cccc}
\hline \hline Parameter & Optimized Value $[\mathrm{mm}]$ & Parameter & Optimized Value [mm] \\
\hline $\mathbf{a}$ & 7.5 & $\mathbf{f}_{\mathbf{2}}$ & 4.2 \\
$\mathbf{b}$ & 7.5 & $\mathbf{s}_{\mathbf{1}}$ & 6.0 \\
$\mathbf{c}$ & 46.6 & $\mathbf{\mathbf { s } _ { \mathbf { 2 } }}$ & 3.4 \\
$\mathbf{\mathbf { f } _ { \mathbf { 1 } }}$ & 3.5 & $\mathbf{L}$ & 60 \\
\hline \hline
\end{tabular}

Furthermore, since the four radiating elements are arranged to form an annular square structure (Fig. 2a), each element is 90degree rotated with respect to the center of the common ground plane. Thus, by feeding each element with currents having the same amplitude, but with a 90-degree phase shift, a circularly polarized radiated field can be achieved (sequential rotation feeding technique) leading to an improvement of both cross polarization (circular polarization purity) and radiation pattern symmetry. Consequently, a specific matching and delay line, capable of driving each radiating element with the required phaseshifted currents, has been designed and placed upside-down with respect to the radiating structures so to share the same ground plane (Fig. 2b). Moreover, as better described in [18], this feeding line, has been optimized to exhibit a standard $50 \Omega$ input impedance when its four ports are connected to each $100 \Omega$ radiating element (i.e. the F-inverted monopoles in the considered structure).

The feeding line equivalent circuit is shown in Fig. 2c, where the indicated microstrip lines characteristic impedances (i.e. microstrip widths) have been conveniently optimized for a 3D-printed $0.85 \mathrm{~mm}$-thick PLA substrate (100\% of infill also in this case). Also, by properly adjusting the $\ell_{i}$ lengths, the microstrip length between two consecutive ports becomes close to $\lambda_{\mathrm{g}} / 4$ (being $\lambda_{\mathrm{g}}$ the equivalent guide wavelength), and a 90-degree-phase shifting among each antenna is obtained. It is worth noting that the size and shape of the 3D-printed supports can be arbitrarily chosen, differently from other commercial substrates whose thickness is standardized.

\section{ANTENNA REALIZATION}

In this Section the manufacturing process to prototype the proposed 3D-printed antenna for handheld UHF RFID readers will be illustrated. This process consists in fabricating the metallic parts and the PLA substrates of both feeding network and radiating 
structure and then in carefully assembling them.

In Fig. 3, different pictures of the realized antenna are reported. In particular, in Fig. 3a, the antenna top view with the four radiating elements placed over an annular square PLA low-loss support can be observed. The bottom view, representing the feeding network, is shown in Fig. 3c. Finally, according to the design strategy of Section II, a common ground plane is foreseen to be shared between radiating elements and feeding network. Fig. 3b shows this ground plane (applied on the second PLA substrate) along with slots for SMA connector and four via-holes for the feeding lines.

From the fabrication point of view, as for the two PLA parts (i.e. the annular square and the feeding network substrates), they have been molded by using a Sharebot NextGeneration 3D printer having a $1.75 \mathrm{~mm}$ filament extruder, a $0.35 \mathrm{~mm}$ nozzle and a $45 \times 45 \mathrm{~cm}^{2}$ heatbed plate. A printing speed as low as $25 \mathrm{~mm} / \mathrm{s}$ for the first layer, (so to facilitate the adhesion between PLA and plate) and at $50 \mathrm{~mm} / \mathrm{s}$ for the rest of the structure has been set along with a plate temperature of $65^{\circ} \mathrm{C}$.

On the other hand, as for the conductive parts (i.e. radiating elements, feeding network, feeding lines, and ground plane), they have been realized by using $35 \mu \mathrm{m}$ adhesive copper tape shaped through the cutting-plotter-based technique presented in [19]. All the conductive parts have been manually applied on the realized 3D structures according to Fig. 3. Finally, the four lateral feeding lines connecting the feeding network to each element of the radiating structure have been applied on the substrate and

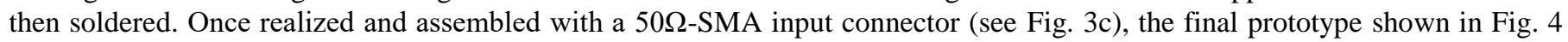
has been obtained.

Fig. 4 Prototype of the 3D-printed antenna for handheld UHF RFID Reader

\section{RESULTS}

In this Section the performance of the antenna have been extensively validated. In Fig. 5, for instance, simulated and measured reflection coefficients and axial ratio are shown and compared.

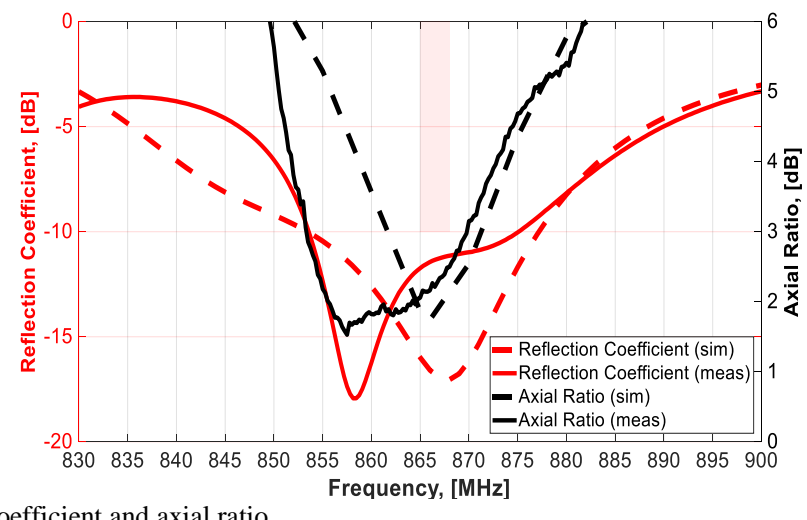

Fig. 5 Simulated and measured reflection coefficient and axial ratio.

It is worth noting that the simulated reflection coefficient assumes values below $-10 \mathrm{~dB}$ in the frequency range $854-877 \mathrm{MHz}$, which covers the required ETSI UHF-RFID band $(865-868 \mathrm{MHz})$. Also, the simulated axial ratio in the boresight direction $\left(\theta=0^{\circ}\right.$ in Fig. 2) is lower than $3 \mathrm{~dB}$ in the frequency range $862-871 \mathrm{MHz}$. The measured reflection coefficient and axial ratio are slightly shifted toward lower frequency due to small variation of the dielectric characteristics considered in the simulated model, but they are still satisfactory in the considered ETSI UHF-RFID band.

The radiation patterns in the $\varphi=0^{\circ}$ and $\varphi=90^{\circ}$ planes, at the frequency of $866.5 \mathrm{MHz}$, are plotted in Fig. 6. In particular, both the copolar (Left Hand Circular Polarization, LHCP) and the crosspolar (Right Hand Circular Polarization, RHCP) field components are plotted as a function of the elevation angle $\theta$ (referred to the coordinates system shown in Fig. 2). A good agreement between simulated and measured copolar (LHCP) radiation pattern is achieved on both the considered planes. The measured crosspolar component (RHCP) is slightly higher than the simulated one, but in the boresight direction $\left(\theta=0^{\circ}\right)$ is still 12 $15 \mathrm{~dB}$ lower than the copolar component, confirming the good axial ratio performance shown in Fig. 5. The measured gain is about $1.5 \mathrm{~dB}$ and it is constant in the whole ETSI UHF-RFID band. 

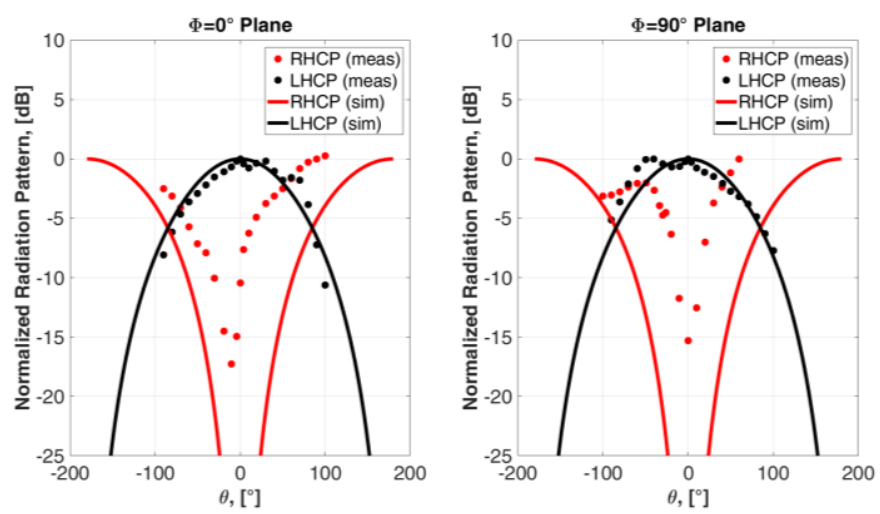

Fig. 6 Simulated (solid line) and measured (markers) radiation pattern in the (a) $\varphi=0^{\circ}$ and (b) $\varphi=90^{\circ}$ planes, at a frequency of $866.5 \mathrm{MHz}$. Both the copolar (LHCP) and crosspolar (RHCP) are plotted.

In order to characterize the antenna also from a system level point of view, further results have been obtained, similarly to the experimental analysis described in [20]. In particular, in Fig. 7, the measurement setup for an RSSI evaluation test is shown. The Antenna Under Test (AUT) has been placed at $1 \mathrm{~m}$ above the ground level and fed with an input power of $150 \mathrm{~mW}$ by an R4300P ION commercial RFID Reader from the CAEN RFID company [21]. The frequency hopping has been enabled in the ETSI UHF RFID band. Microwave absorbers have been used to mitigate the reflection from the ground. A dipole-like UHF RFID tag (Alien 9640) has been placed at a variable distance from the reader antenna, attached on a thin wood pole at $1 \mathrm{~m}$ above the ground.

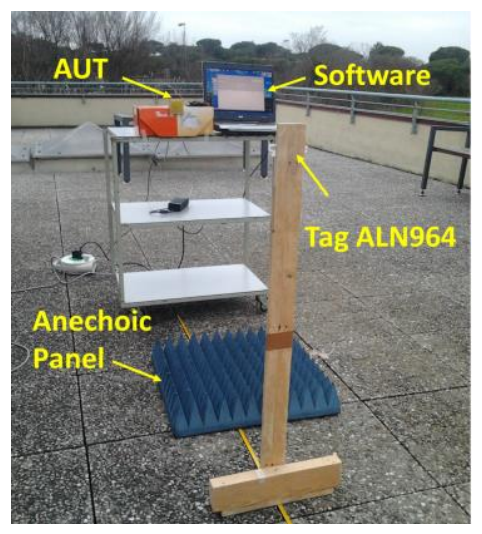

Fig. 7 Measurement setup for the read range test.

The measured RSSI as a function of the tag-reader distance and of tag orientation is shown in Fig. 8. In particular, in this plot the performance of the proposed 3D printed antenna is compared with the single band double-layer RFID Reader antenna considered in [20]. The latter is a low-cost version of the single-band circularly polarized antenna presented in [7] and optimized for the ETSI UHF RFID ( $865-868 \mathrm{MHz}$ ). Its size is $60 \times 60 \times 7 \mathrm{~mm}^{3}$, comparable with the 3D printed antenna, and it is composed by two low-cost FR-4 $\left(\varepsilon_{\mathrm{r}}=4.3\right.$, $\left.\tan \delta=0.025\right)$ substrates arranged in a stacked configuration. It is worth noting that the here proposed low-cost 3D-printed antenna is able to detect the ALN 9640 tag up to $180 \mathrm{~cm}$ of distance, independently from its orientation. On the other hand, the double-layer antenna can detect tags up to $150 \mathrm{~cm}$ with the same input power. This confirms that, by exploiting the 3D printed structure, the proposed antenna guarantees a performance improvement without exceeding the limited available volume. In conclusion, the here proposed 3D-printed antenna is able to guarantee a satisfactory read range while maintaining a low-profile and compact structure. Thanks to the 3D printing, the overall antenna is light, easy to fabricate and low-cost, differently from other configurations including double-layer structures, as in [7], [17]. Nevertheless, by properly optimizing the geometry of the 3D-printed structure, it may be even possible to further improve the antenna performance, especially in terms of gain. 


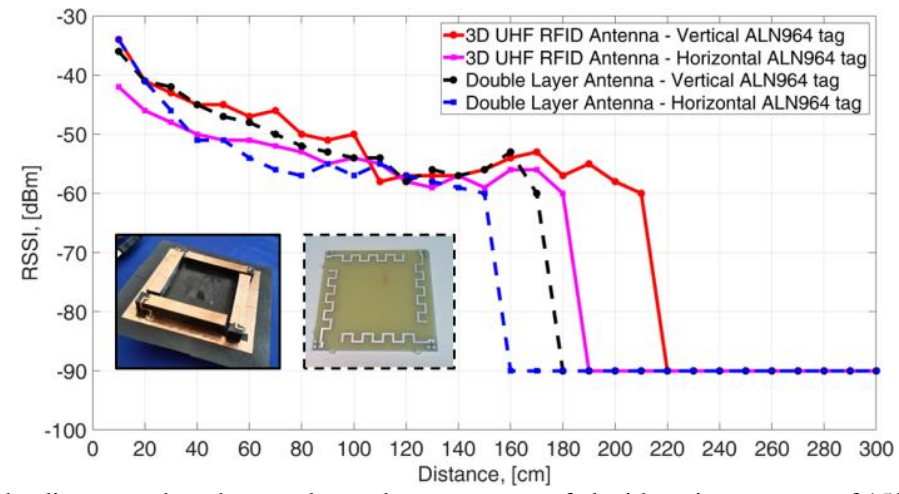

Fig. 8 Measured RSSI as a function of the distance, when the portabe reader antennas are fed with an input power of $150 \mathrm{~mW}$

\section{CONCLUSIONS}

In this paper, Additive Manufacturing 3D-printing technology has been exploited to design a circularly polarized antenna for ETSI UHF-RFID handheld readers. The antenna consists of four inverted-F monopoles with capacitive top loads, sharing a single ground plane which is in common also with a feeding network mounted upside-down. The feeding network, in turn, implements the sequential rotation feeding technique to achieve a circularly polarized radiated field. As demonstrated by simulated and experimental results, thanks to 3D-printed supporting structures, specifically tailored and realized in Poly-Lactic Acid, a limited antenna size is obtained while guaranteeing performance superior to other compact solutions proposed in the literature and based on conventional materials.

\section{REFERENCES}

[1] S. Jun, B. Sanz-Izquierdo, E.A. Parker, "3d Printing Technique for the Development of Non-Planar Electromagnetic Bandgap Structures for Antenna Applications," Electronics Letters, 2016, 52, (3), pp. 175-180.

[2] S.S. Patel, I.J.G Zuazola, and W.G. Whittow, “Antenna with Three Dimensional 3d Printed Substrates," Microwave and Optical Technology Letters, 58, (4), pp. 741-744, 2016.

[3] S. Moscato, R. Bahr, T.R. Le, M. Pasian, M. Bozzi, L. Perregrini, and M.M. Tentzeris, "Infill-Dependent 3-D-Printed Material Based on Ninjaflex Filament for Antenna Applications," IEEE Antennas and Wireless Propagation Letters, 2016, 15, pp. 1506-1509.

[4] Nasimuddin, Z. N. Chen, and X. M. Qing, "Asymmetric-Circular Shaped Slotted Microstrip Antennas for Circular Polarization and RFID Applications," IEEE Trans. on Antennas and Propagation, vol. 58, pp. 3821-3828, Dec 2010.

[5] Mirchandani, P., Deshpande, K., Khan, R. "Performance analysis of miniaturized microstrip patch antennas for UHF RFID applications" (2011) 2011 International Conference on Signal Processing, Communication, Computing and Networking Technologies, ICSCCN-2011, art. no. 6024552, pp. $244-249$.

[6] Y. F. Lin, Y. K. Wang, H. M. Chen, and Z. Z. Yang, "Circularly Polarized Crossed Dipole Antenna With Phase Delay Lines for RFID Handheld Reader," IEEE Trans. on Antennas and Propagation, vol. 60, pp. 1221-1227, Mar 2012.

[7] J. H. Bang, C. Bat-Ochir, H. S. Koh, E. J. Cha, and B. C. Ahn, "A Small and Lightweight Antenna for Handheld RFID Reader Applications," IEEE Antennas and Wireless Propagation Letters, vol. 11, pp. 1076-1079, 2012.

[8] Nasimuddin, X. M. Qing, and Z. N. Chen, "Compact Circularly Polarized Microstrip Antenna for RFID Handheld Reader Applications," Apmc: 2009 Asia Pacific Microwave Conference, Vols 1-5, pp. 1950-1953, 2009.

[9] Nasimuddin, Chen, Z.N., Qing, X., "Compact circularly polarized asymmetric-slotted microstrip patch antennas" (2012) Microwave and Optical Technology Letters, 54 (8), pp. 1920-1927.

[10] Nasimuddin, Qing, X., Chen, Z.N. "Compact asymmetric-slit microstrip antennas for circular polarization" (2011) IEEE Trans. on Antennas and Propagation, 59 (1), art. no. 5617246, pp. 285-288.

[11] Nasimuddin, Chen, Z.N., Qing, X. "Compact arc-shaped slotted circularly polarized microstrip antenna for RFID readers" (2011) Final Program and Book of Abstracts - iWAT 2011: 2011 IEEE International Workshop on Antenna Technology: Small Antennas, Novel Structures and Innovative Metamaterials, art. no. 5752312, pp. 340-343.

[12] S. A. Yeh, H. M. Chen, Y. F. Lin, C. Kao, and J. Y. Jan, "Single-Layer Circularly Polarized Slot Antenna for RFID Reader Application," 2010 IEEE Antennas and Propagation Society International Symposium, 2010. 1522-3965.

[13] S. A. Yeh, Y. F. Lin, H. M. Chen, and J. S. Chen, "Slotline-Fed Circularly Polarized Annular-Ring Slot Antenna for RFID Reader," Apmc: 2009 Asia Pacific Microwave Conference, Vols 1-5, pp. 618, 2009.

[14] Y. F. Lin, H. M. Chen, F. H. Chu, and S. C. Pan, "Bidirectional radiated circularly polarised square-ring antenna for portable RFID reader," Electronics Letters, vol. 44, pp. 1383-U1, Nov 202008.

[15] S. Kim, H. Park, D. Lee, J.Choi, "A novel design of an UHF RFID reader antenna for PDA," Apmc: 2006 Asia Pacific Microwave Conference, APMC, Vol. 3, pp. 1471-1473, 2006.

[16] Y. F. Lin, H. M. Chen, S. C. Pan, Y. C. Kao, and C. Y. Lin, "Adjustable axial ratio of single-layer circularly polarised patch antenna for portable RFID reader," Electronics Letters, vol. 45, pp. 290-291, Mar 122009.

[17] R. Caso, A. Michel, M. Rodriguez-Pino and P. Nepa, "Dual-Band UHF-RFID/WLAN Circularly Polarized Antenna for Portable RFID Readers," in IEEE Trans. on Antennas and Propagation, vol. 62, no. 5, pp. 2822-2826, May 2014.

[18] L. Catarinucci, R. Colella, P. Coppola and L. Tarricone, "Microwave characterisation of polylactic acid for 3D-printed dielectrically controlled substrates," in IET Microwaves, Antennas \& Propagation, vol. 11, no. 14, pp. 1970-1976, 1119 2017. Ü

[19] L. Catarinucci, R. Colella, and L. Tarricone, "Smart Prototyping Techniques for UHF RFID Tags: Electromagnetic Characterization and Comparison with Traditional Approaches," Progress in Electromagnetics Research, vol. 132, pp. 91-111, 2012.

[20] R. Caso, A. Michel, P. Nepa and M. R. Pino, "Performance comparison between an UHF RFID antenna for portable reader and its UHF RFID/WLAN dualband version," 2014 XXXIth URSI General Assembly and Scientific Symposium (URSI GASS), Beijing, 2014, pp. 1-4.

[21] http://www.caenrfid.it/en/ 